\title{
TITLE:
}

\section{Copper nitride thin films prepared by radio-frequency reactive sputtering}

$\operatorname{AUTHOR}(S)$ :

MARUYAMA, T; MORISHITA, T

CITATION:

MARUYAMA, T ... [et al]. Copper nitride thin films prepared by radiofrequency reactive sputtering. JOURNAL OF APPLIED PHYSICS 1995, 78(6): 4104-4107

ISSUE DATE:

1995-09-15

URL:

http://hdl.handle.net/2433/43537

\section{RIGHT:}

Copyright 1995 American Institute of Physics. This article may be downloaded for personal use only. Any other use requires prior permission of the author and the American Institute of Physics. 
Toshiro Maruyama and Tomonori Morishita

Department of Chemical Engineering, Faculty of Engineering, Kyoto University, Yoshida-Honmachi, Sakyo-ku, Kyoto 606, Japan

(Received 28 April 1995; accepted for publication 7 June 1995)

\begin{abstract}
Copper nitride thin films were obtained by the reactive sputtering method. A metallic copper target was sputtered in nitrogen gas with radio-frequency (rf) magnetron sputtering equipment. Highly [100]-oriented polycrystalline films of the cubic anti- $\mathrm{ReO}_{3}$ structure were obtained. Films with a lattice constant above $3.868 \AA$ were conductors, while films with a lattice constant below $3.868 \AA$ were insulators. The resistivity of conducting films was $0.5-3 \times 10^{-2} \Omega \mathrm{cm}$. The insulating films showed an optical energy gap of $1.3 \mathrm{eV}$, while the conducting films showed a smaller value which decreased with decreasing resistivity. (-) 1995 American Institute of Physics.
\end{abstract}

\section{INTRODUCTION}

Copper nitride $\left(\mathrm{Cu}_{3} \mathrm{~N}\right)$ has the cubic anti- $\mathrm{ReO}_{3}$ structure. Since $\mathrm{Cu}$ atoms do not occupy the close-packing site of the (111) plane, insertion of $\mathrm{Cu}$ or another atom into the body center of the anti- $\mathrm{ReO}_{3}$ structure is possible. That is, $\mathrm{Cu}_{3} \mathrm{~N}$ has the possibility (in applications) to be an inorganic host material like $\mathrm{WO}_{3}$, which makes conductive tungsten bronzes by inserting some metal atoms into the $\mathrm{ReO}_{3}$ structure. However, all the $\mathrm{Cu}_{3} \mathrm{~N}$ films reported ${ }^{1-4}$ have been insulators. In addition, $\mathrm{Cu}_{3} \mathrm{~N}$ is stable at room temperature, while the thermal decomposition takes place at about 350 $450^{\circ} \mathrm{C}$. Previous studies have been mainly concerned about the applications of its low decomposition temperature. Asano et al. ${ }^{1}$ prepared the films by ion-assisted vapor deposition, and made preliminary experiments of the write-once optical recording on this film using the heat mode. Maya ${ }^{2}$ obtained the films by dc sputtering, and explored the feasibility of using the coatings to generate microscopic metal lines by maskless laser writing. ${ }^{3}$

Our objective is to prepare copper nitride thin films by using reactive if magnetron sputtering of a copper target. The sputtering parameters used were substrate temperature, if power, and total pressure. The electrical and optical characteristics are clarified, and the effects of deposition conditions on the measured structure of the film are discussed.

\section{EXPERIMENT}

In preparing copper nitride films, if (13.5 MHz) magnetron sputtering equipment (Osaka Vacuum Co. Ltd.) ${ }^{5,6}$ was used with a $99.99 \%$ pure copper target of $10 \mathrm{~cm}$ in diameter and $1 \mathrm{~mm}$ thick. The rf power was $20-400 \mathrm{~W}$, the sputtering gas was $99.999 \%$ pure nitrogen, and its flow rate ranged 10-200 sccm. The separation distance between the substrate and the target was $48 \mathrm{~mm}$. The chamber vacuum just before growth was less than $2.0 \times 10^{-6}$ Torr. The substrate was heated by a lamp heater to a temperature in the range 50$300^{\circ} \mathrm{C}$. It was measured using a chromel-alumel thermocouple attached to the front of the substrate holder. During the run at a substrate temperature below $200^{\circ} \mathrm{C}$, the temperature increased because of the effect of plasma heating. Since the rate decreased with increasing time, the final substrate temperature was used as a representative substrate temperature. The total sputtering pressure ranged from 0.76 to 28 mTorr. A $76 \times 26 \mathrm{~mm}^{2}$ borosilicate glass plate was used as the substrate.

The crystallinity of the film was analyzed by the $x$-ray diffraction (XRD) method (Shimadzu XD 610) with $\mathrm{Cu} K \alpha$ radiation. The electric resistivity of the film was measured by the van der Pauw method. The carrier concentration and Hall mobility were measured by using the Hall effect. The optical transmittance of the film was obtained by means of a multipurpose recording spectrophotometer. A blank glass substrate was inserted into the reference beam path of the spectrophotometer.

\section{RESULTS AND DISCUSSION}

Smooth copper nitride films were formed, which were transparent and colored dark brown on the glass substrate. Figure 1 shows the deposition rate as a function of if power.

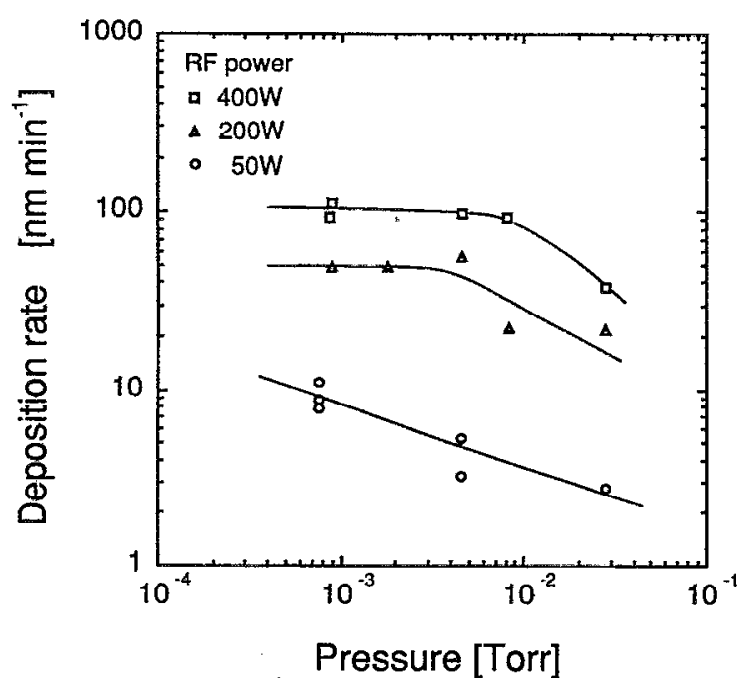

FIG. 1. Deposition rate as a function of rf power and total pressure. 


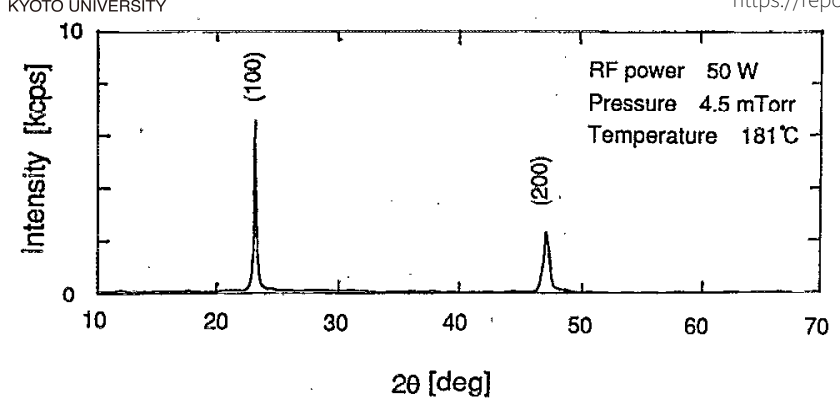

FIG. 2. X-ray diffraction pattern of 133-nm-thick film on a borosilicate glass substrate.

and total pressure. A similar dependence on total pressure was observed in depositing $\operatorname{InN}$ and $\mathrm{SnN}_{x}$ films. ${ }^{5,6}$ Visual observations of the deposition at high total pressure showed that the surface of the target was covered with a black nitrided layer after each run.

Figure 2 shows a typical example of the $\mathrm{x}$-ray diffraction pattern of the film on a glass substrate. The precise (100) and (200) peaks indicate that the film is composed of polycrystallites with a cubic anti- $\mathrm{ReO}_{3}$ structure. A large peak of (100) indicates that the film is highly [100]-oriented. All samples showed similar $\mathrm{X}$-ray diffraction patterns, indicating the highly [100]-oriented cubic anti- $\mathrm{ReO}_{3}$ structure. The crystallinity strongly depends on deposition conditions. Below, the dependence is discussed by using the full width at half-maximum (FWHM) and integrated intensity of the diffraction peak corresponding to a (100) reflection.

Figures 3-5 show the FWHM and integrated intensity as functions of substrate temperature, total pressure, and if power. The films were prepared by keeping two of the following three parameters constant: total pressure of 4.5 mTorr, rf power of $50 \mathrm{~W}$, and a substrate temperature in a range of $50-140^{\circ} \mathrm{C}$ which was the lowest substrate temperature under the effect of plasma heating. Comparisons of integrated intensity were made between films of nearly equal value of the thickness of film. Figure 3 shows that the integrated intensity increases with increasing substrate tempera-

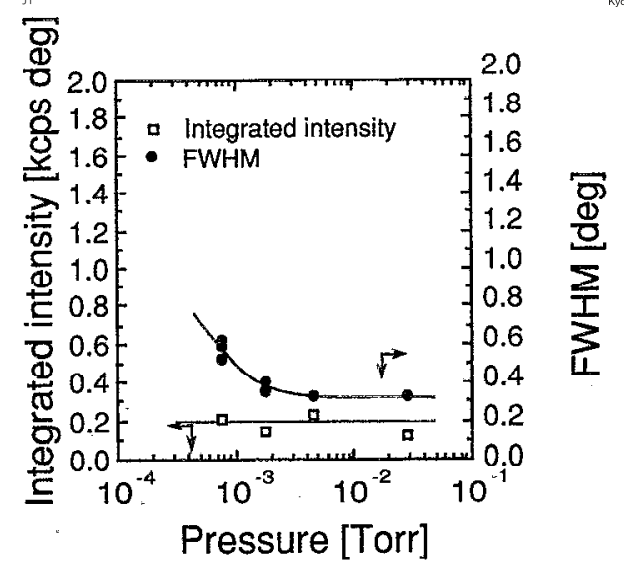

FIG. 4. FWHM and integrated intensity as a function of total pressure. Films were prepared at constant rf power $(50 \mathrm{~W})$ and substrate temperature in a range of $50-140^{\circ} \mathrm{C}$. Film thickness is in the range $78-97 \mathrm{~nm}$.

ture, because of an enhanced surface diffusion of the adatóms at higher substrate temperatures. ${ }^{6}$ At substrate temperatures above $220^{\circ} \mathrm{C}$, however, the integrated intensities show very small values. In addition, the film was amorphous at $300^{\circ} \mathrm{C}$. These are attributable to lattice defects caused by surface adatom reemissions: i.e., the reevaporation of atomic nitrogen from the substrate at higher substrate temperatures. ${ }^{5,6}$ Figure 5 shows that the films of inferior crystallinity are prepared at higher if powers, where the deposition rates show larger values.

For the electrical characteristics, not all the films were insulators, and some films of inferior crystallinity were conductors. For example, some films prepared at substrate temperatures below $100^{\circ} \mathrm{C}$ were conductors, although the films prepared at substrate temperatures above $160^{\circ} \mathrm{C}$ were invariably insulators. In fact, Fig. 3 shows that the films have inferior crystallinities at substrate temperatures below $100^{\circ} \mathrm{C}$. Figure 6 shows the insulator-conductor boundary of deposition conditions for the films prepared at substrate temperatures below $100^{\circ} \mathrm{C}$. A comparison of Fig. 6 with Figs. 4

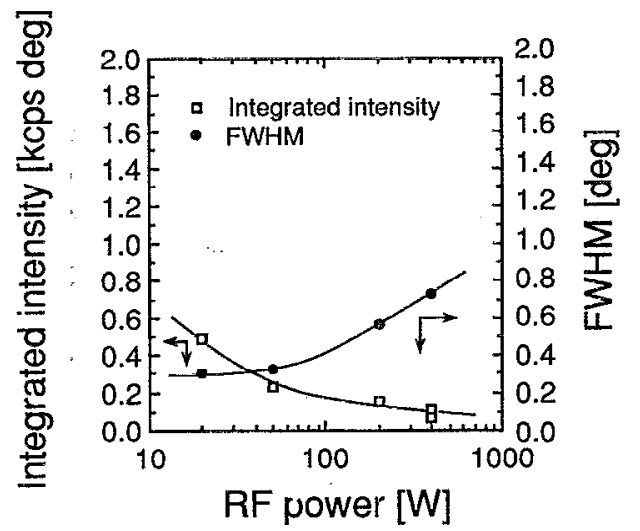

FIG. 5. FWHM and integrated intensity as a function of if power. Films were prepared at constant total pressure $(4.5 \mathrm{mTorr})$ and substrate temperature in a range of $50-140^{\circ} \mathrm{C}$. Film thickness is in the range $97-147 \mathrm{~nm}$.
FIG. 3. FWHM and integrated intensity as a function of substrate temperature. Films were prepared at constant total pressure $(4.5 \mathrm{mTorr})$ and constant If power $(50 \mathrm{~W})$. Film thickness is in the range $88-133 \mathrm{~nm}$. 


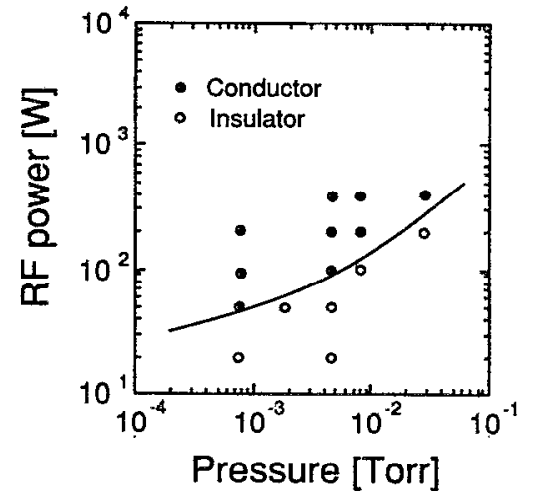

FIG. 6. Insulator-conductor boundary of deposition conditions for films prepared at substrate temperatures below $100^{\circ} \mathrm{C}$.

and 5 shows that the conditions for obtaining conducting films correspond to those for the films of inferior crystallinity.

Figure 7 shows examples of the absorption coefficients as a function of photon energy. The optical energy gap Eg for indirect transitions is obtained by extrapolating the full linc to the abscissa. The value of Eg for the insulator is $1.3 \mathrm{eV}$, while the conductor shows a smaller value which decreases with decreasing resistivity.

The electric conductivity is inferred to be due to an insertion of $\mathrm{Cu}$ atoms into the body center of the anti- $\mathrm{ReO}_{3}$ structure, like $\mathrm{WO}_{3}$, which makes conductive tungsten bronzes by inserting some metal atoms into the $\mathrm{ReO}_{3}$ structure. That is, the inserted $\mathrm{Cu}$ atoms becomes a donor which releases a free electron as a carrier. Therefore, it is inferred that the difference between insulator and conductor is reflected by the differences in lattice constant for inserting $\mathrm{Cu}$ atoms.

Figures 8-10 show the lattice constant as functions of substrate temperature, total pressure, and if power. The results in Fig. 8 were obtained at an if power of $50 \mathrm{~W}$, the results in Figs. 9 and 10 at a substrate temperature in a range of $50-100^{\circ} \mathrm{C}$, and the results in Fig. 10 at a total pressure of

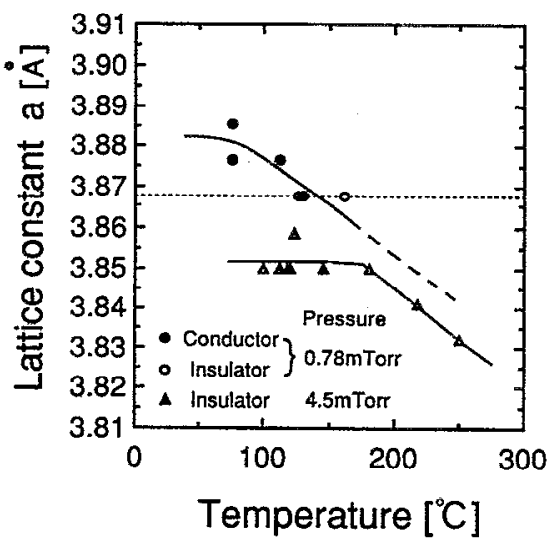

FIG. 8. Lattice constant as a function of substrate temperature.

4.5 mTorr. They are larger than that for powder $(3.815 \AA)$. The lattice constant increases with decreasing substrate temperature, decreasing total pressure, and increasing if power. These conditions correspond to those for obtaining the films of inferior crystallinity, and consequently to those for obtaining the conducting films. Furthermore, the latter conditions can be expressed by using the critical value of a lattice constant. That is, the films with a lattice constant above $3.868 \AA$ are conductors, while the films with a lattice constant below $3.868 \AA$ are insulators.

Figure 11 shows the resistivity, Hall mobility, and carrier concentration for the conducting films. The results obtained at various deposition conditions are correlated as a function of lattice constant. The resistivity is $0.5-3 \times 10^{-2} \Omega \mathrm{cm}$. The carrier concentration does not increase with increasing lattice constant. This fact suggests that the increase in lattice constant is not due to the increase in carrier concentration, i.e., the insertion of $\mathrm{Cu}$ atoms. That is, the film with a lattice constant above the critical value $3.868 \AA$ enables the insertion of a certain number of $\mathrm{Cu}$ atoms. The number of atoms is irrelevant to the increase of lattice constant.

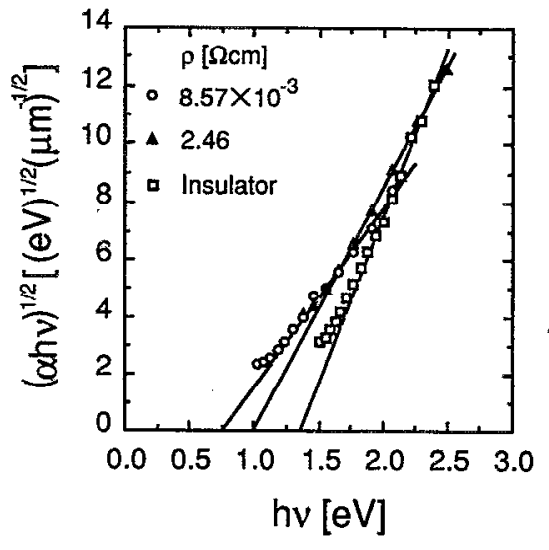

FIG. 7. Relation between absorption coefficient and energy of incident photons.

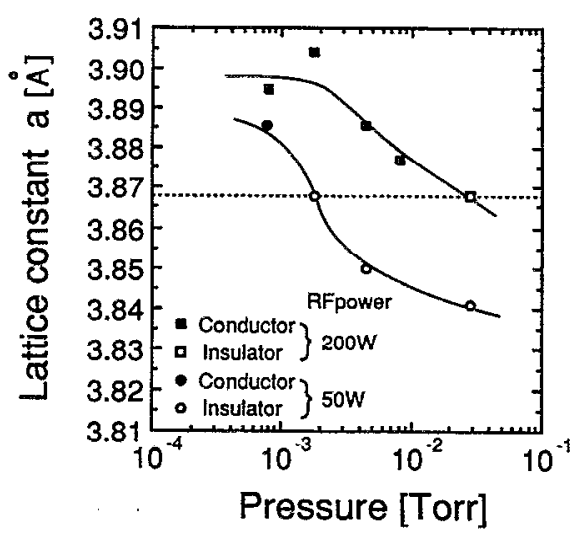

FIG. 9. Lattice constant as a function of total pressure. 


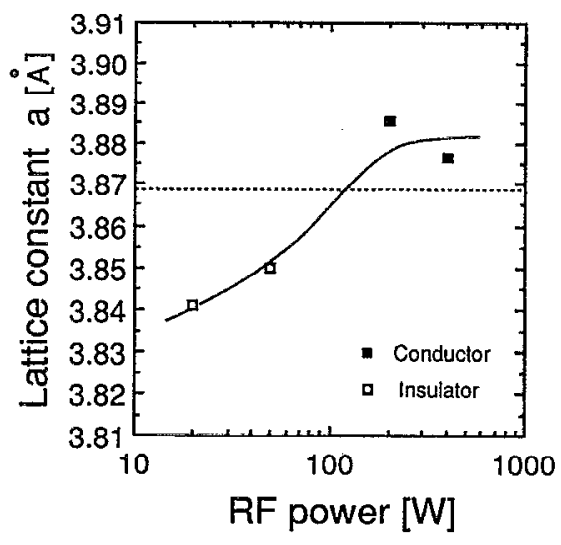

FIG. 10. Lattice constant as a function of if power.

\section{CONCLUSIONS}

Copper nitride thin films were obtained by the reactive sputtering method. The metallic copper target was sputtered in nitrogen gas with if magnetron sputtering equipment. Highly [100]-oriented polycrystalline films of the cubic anti- $\mathrm{ReO}_{3}$ structure were obtained. Films with a lattice constant above $3.868 \AA$ were conductors, while films with a lattice constant below $3.868 \AA$ were insulators. The resistivity of conducting films was $0.5-3 \times 10^{-2} \Omega \mathrm{cm}$. Insulating films showed an optical energy gap of $1.3 \mathrm{eV}$, while conducting films showed a smaller value which decreases with decreasing resistivity.

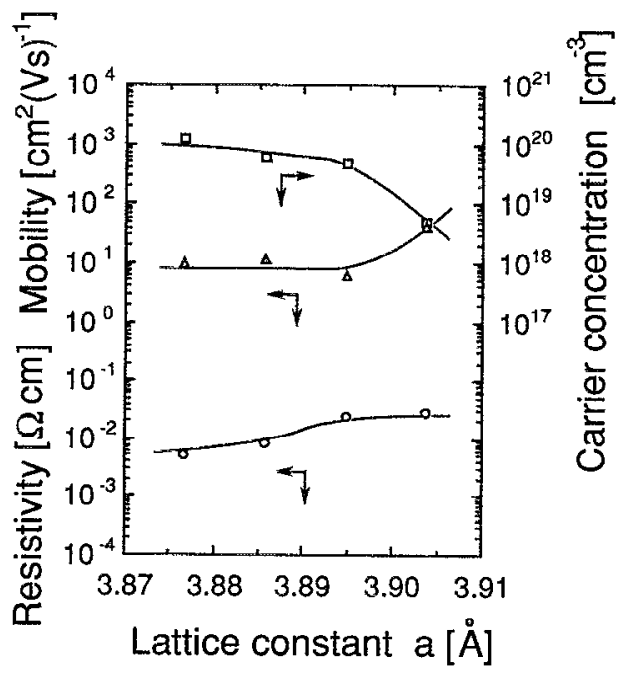

FIG. 11. Resistivity, Hall mobility, and carrier concentration as a function of lattice constant.

\section{ACKNOWLEDGMENTS}

This work was supported by the Ookura Foundation. The authors thank T. Yamaoka of Nippon Sheet Glass Co., Ltd., for the Hall coefficient measurements.

${ }^{1}$ M. Asano, K. Umeda, and A. Tasaki, Jpn. J. Appl. Phys. 29, 1985 (1990).

${ }^{2}$ L. Maya, J. Vac. Sci. Technol. A 11, 603 (1993).

${ }^{3}$ L. Maya, Mater. Res. Soc. Symp. Proc. 282, 203 (1993).

${ }^{4}$ S. Terada, H. Tanaka, and K. Kubota, J. Cryst. Growth 94, 567 (1989).

${ }^{5}$ T. Maruyama and T. Morishita, J. Appl. Phys. 76, 5809 (1994).

${ }^{6}$ T. Maruyama and T. Morishita, J. Appl. Phys. 77, 6641 (1995). 